\title{
Enhancing Learning Multimodality: A Reflection of Audio-Visual Media Use in EFL Classes
}

\author{
Paulus Widiatmoko, Universitas Kristen Duta Wacana \\ Ignatius Tri Endarto, Universitas Kristen Duta Wacana
}

\begin{abstract}
Text-based material is known to deliver merely visual messages, unlike audio-visual-media which offer both visual and auditory signals for learners to decode. Meanwhile, practicing English as a foreign language requires sufficient exposures to language input, both auditory and visual. In this study, principles of multimedia learning and multimedia processing for material design are chosen as its initial frameworks. Then, samples of practice material and media implemented in the researchers' EFL classes are discussed in the light of how multimodalities were accommodated. In this study, three classes of English for Specific Purposes used audiovisual media to offer more multimodal exposures in terms of learning contexts, target culture understanding, and language function practice. In reflection, it is concluded that audio-visual media provide promising potentials to empower teachers, engage and motivate learners, and eventually facilitate language learning.
\end{abstract}

Keywords: audio; EFL; multimodality; visual

\section{Introduction}

Human communication is multimodal. People converse using more than verbal language. They embrace 3D properties by paying attention to gestures and facial expressions accompanied by intonation and word stress. In fact, contextual and cultural aspects of the discourse also enrich the communication multimodality.

In the context of teaching and learning a foreign language, features of non-verbal modalities, therefore, should be included. Replication of authentic communication along with its rich multimodality needs to be presented for the students to learn. Textual material alone has limitation of depicting real situation of communication due to the lack of audio and 3D visual of interlocutors participating.

With regard to students' learning styles which may vary from one another, traditional print-based material may not always be the best solution, especially for those who prefer to learn auditorily. Hence, integrating more modes into one's teaching would broaden the scope for accommodating learning style diversities. To that end, this study aims at scrutinizing the use of audio-visual media to assist learning multimodality in EFL classes.

\section{Multimodality and Language Learning}

All types of learning, including language learning, entail certain cognitive processing which can always be facilitated through multimodality. As explained earlier, it is all due to the fact that human communication aka language itself is multimodal. Even in natural language learning, children tend to start imitating gestures and facial expressions before they learn to utter words. A study conducted by Matthiessen (2017) has been able to show that gestures are indeed used in association with other modes of expression such as words to create meaning. This indicates that multimodality is inherent in both language and language learning. Given that the popularity of digital interactive media has enabled teachers to bring various modes of communication into the classroom, it is not overrating to state that multimodality should be considered an integral part of material development for today's language learning.

But what is multimodality? It is actually a term that stems from the field of social semiotics. According to Kress (2010: 22) multimodality is "representations in many modes, each chosen from rhetorical aspects for its communicational potentials". Fortanet-Gómez \& 
Camiciottoli (2015: 1) on the other hand see multimodality as an approach to communication studies, by stating that it is "an approach used to understand the contribution of various semiotic resources (e.g., verbal, visual, aural, spatial)". Both views however adopt the same concept of using multiple modes to serve human communicative purposes.

In order to reflect on how multimodality could facilitate language learning, it is crucial to find out how linguistic systems influence the whole process of multimodal communication in the first place. According to Tang (in Fortanet-Gómez \& Camiciottoli, 2015: 5), one of the wellknown models describes multimodal communication as a process that comprises 5 stages: system of possible semiotic modes, genres, thematic patterns, multimodal instance, and interpretation.

Figure 1: Adapted model of Tang's generalized multimodal instantiation hierarchy (FortanetGómez \& Camiciottoli, 2015: 5)

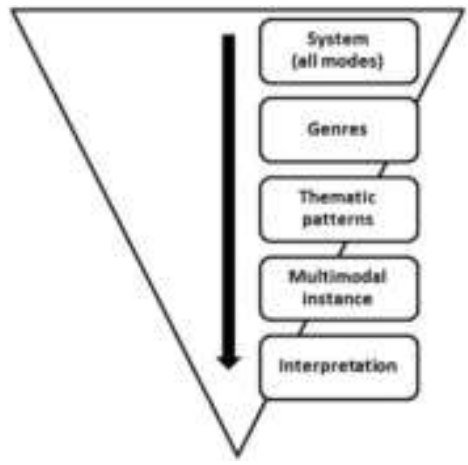

In the first stage of multimodal communication, meaning is generated from the linguistic system through the combination of semiotic modes possible within a culture. It is then followed by the next stage in which meaning is processed via genres that are possible in those modes within that culture. The third stage in order is thematic patterns which underpin the communication. Then, multimodal instance or the actual example of the communication comes fourth. And finally, the messages are interpreted by receivers, or in this case language learner.

For learning to take place and make the best use of multimodality, one needs to consider how cognitive processing actually transpires. This has been scrutinized by Mayer (2009: 200) in his modality principle: "people learn more deeply from pictures and spoken words than from pictures and printed words". The principle is applicable when three conditions are met: the information is complex, the material presentation is fast-paced, and the learners are both fluent and familiar with the language. However, he further claims that in the case of EFL learners who are not familiar with the words, on-screen text or printed words might help. More detailed discussion on how this principle relates to students' learning will be elaborated in the forthcoming sections.

\section{Multimedia and Language Learning}

Another term that is closely related to and perhaps even the best match for multimodality is what is known as multimedia. It could be defined as the combination of a variety of communication channels into a co-ordinated communicative experience for which an integrated cross-channel language of interpretation does not exist by Elsom-Cook (in Mishra \& Sharma, 2005). According to Reddi (2003) proposes that multimedia is an integration of multiple media elements (audio, video, graphic, text, animation, etc.) into one synergic and symbiotic whole that results in more benefits for the end users than any one of the media elements can provide individually. Both propose multimedia as a combination of various media 
and types of communication to bring more benefits than one of them can offer. It has been acknowledged that multimedia has been widely elaborated with more traditional methods of education such as lectures and tutorials, especially to address clearer illustration of complex objects, accommodating different learning styles, improving retention and recalling memories, and facilitating non-verbal learners by Wright (in Mishra \& Sharma, 2005).

Scholars propose that multimedia has been acknowledged to facilitate language learning. First, it is claimed to facilitate different modalities of learning, one of which is the coding of verbal and non-verbal communication as stated (in Berk, 2009). Similar to real life conversation, these modes of communication inevitably interact. Non-verbal clues through body language or facial expression play very essential roles of completing information of verbal language. Other clues depicting context and visual information from a piece of communication discourse in a video scene also complements the verbal language, eventually facilitating comprehension. Secondly, dual channel assumption (Baddeley, 1986) and Paivio (in Mishra, 2005) propose two distinct channels in human cognitive system for representing and manipulating knowledge: a visual pictorial channel and an auditory verbal channel. Thus, moving pictures in videos enter the cognitive system through the eyes and may be processed as pictorial representations in the visual pictorial channel. Spoken words enter the cognitive system through the ears and may be processed as verbal representation in the auditory verbal channel. Then, active processing assumption which states that meaningful learning occurs when learners engage in active processing within the channels, including selecting relevant words and pictures, organizing them into coherent pictorial and verbal models, and integrating them with each other and with appropriate prior knowledge by Mayer (1999); Wittrock (1989, in Mayer, 2009)

Figure 2: Cognitive theory of multimedia learning (Mayer, 2009)

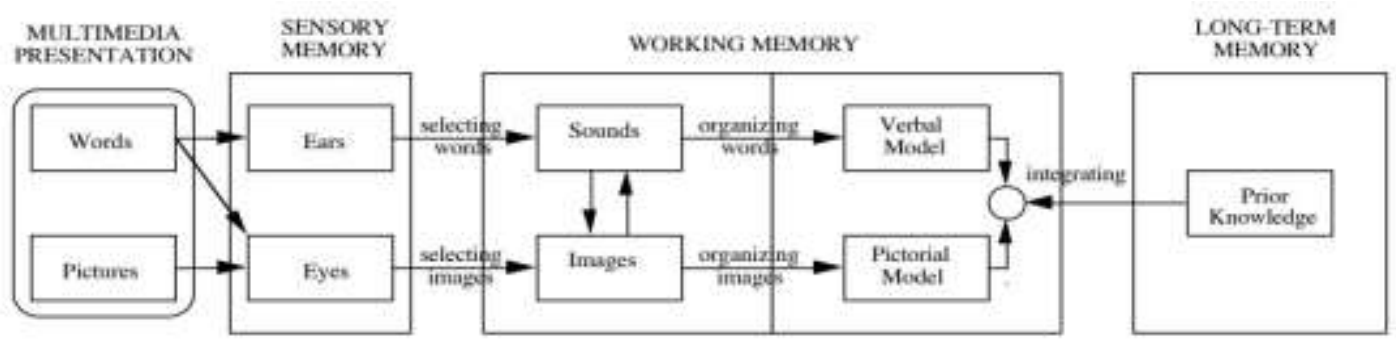

\section{Audio-Visual Media and Multimodal Learning}

In line with O'Halloran (2004: 1), language is no longer seen as an isolated phenomenon separate from visual/gestural codes. In fact both verbal and visual elements have, more often than not, become interdependent in day-to-day communication. Language becomes contextualized when verbal code is accompanied by other semiotic codes, and learners certainly need to be exposed to such a contextualized type of communication. This need for a more multimodal language exposure can be well facilitated by audio-visual media.

As Bruti (2015: 217) puts it, one of the benefits offered by audio-visual texts in the contexts of EFL learning is "that they offer a contextualized representation of communication in which the verbal code is paralleled and complemented by other semiotic codes". In traditional listening activities, learners are forced to comprehend materials only with their ears. Audiovisual media on the other hand alleviate their difficulties by visualizing the messages, and hence making sense of the saying "a picture is worth thousand words". If planned well, they can bring motivation and excitement into the classroom via authentic language by Tomalin ( in Bajrami \& 
Ismaili, 2016). Speaking of authenticity, the media become even more relevant to the EFL contexts by not only displaying real life situations but also providing learners with exposures to native speakers' or other ESL speakers' speech.

There has been a large number of studies of audio-visual media use in EFL settings, few of which are Al-Khayyat (2016), Ashaver and Igyuve (2013), Bajrami and Ismaili (2016), Rezaie and Barani (2011), and Rusmiati and Rosdiana (2017). Though audio-visual media can be classified in several different ways, they basically include film and filmstrips, videotapes, TV programs, dramatization, demonstration, educational programs/games, field trips, etc. (Ashaver \& Igyuve, 2013: 48). This study focuses on the use of online videos in English for Specific Purposes (ESP) classes.

\section{Implementing Audio-Visual Media for ESP Classes}

Audio-visual media for three ESP classes were taken as the data of this study. Those classes are English for Job Interview, English for Tropical Medicine, and English for Presentation. They aim at equipping students with the English skills necessary for their professional lives. Prior to their implementation, videos for job interview simulations, medical procedures of patient examination, and presentation tutorials from Youtube were classified. They were then presented on a website with direct links to Youtube for easier access and convenient use of video streaming features.

A deeper reflection on the design and organization of the audio-visual media in those three classes is elaborated further through the principles of multimedia learning proposed by Mayer (2014). Five principles were emphasized in this study, namely: coherence, signaling, redundancy, spatial contiguity, and temporal contiguity.

\subsection{Coherence Principle}

According to this principle, people learn more deeply from multimedia messages when extraneous material is excluded rather than included. People focus on the essential material better if erroneous material potentials for distractions is eliminated. The media in this study are presented on a website containing links to stream Youtube videos. No changes could be made in relation to the content or selection of its parts due to limitation of these facilities on Youtube. However, all the videos on the website were carefully selected and categorized based on specific topics and subtopics to minimize the inclusion of irrelevant materials.

Figure 3: The videos were selected and organized based on specific topics and subtopics

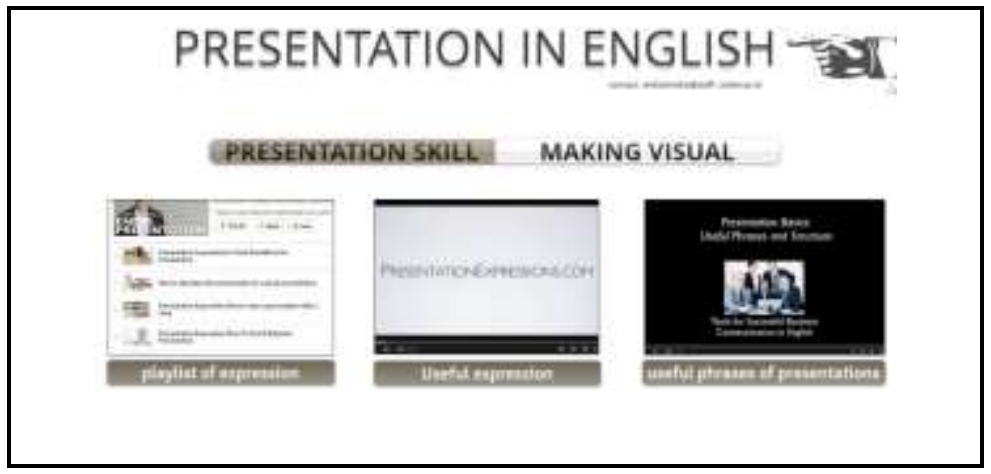

During implementation of the media in the classrooms, teachers may also ensure the coherence principle by selecting relevant and necessary scenes from the videos to make learning 
more focused. Parts of videos that are not essential for meeting the learning objectives could be skipped.

\subsection{Signaling Principle}

The signaling principle states that people learn more deeply from a multimedia message when cues are added highlighting organization of the essential material. Rationally, people learn better if the lesson is designed to call their attention to the important material and how it is organized. Similar with the previous one, this principle is encouraged by means of teacher strategies at the time of media implementation in the class. For instance, some job interview videos on Youtube provide narration as introduction to the topics or display keywords for the students to focus on the essential points.

Figure 4: Cues to highlight the organization of the essential material

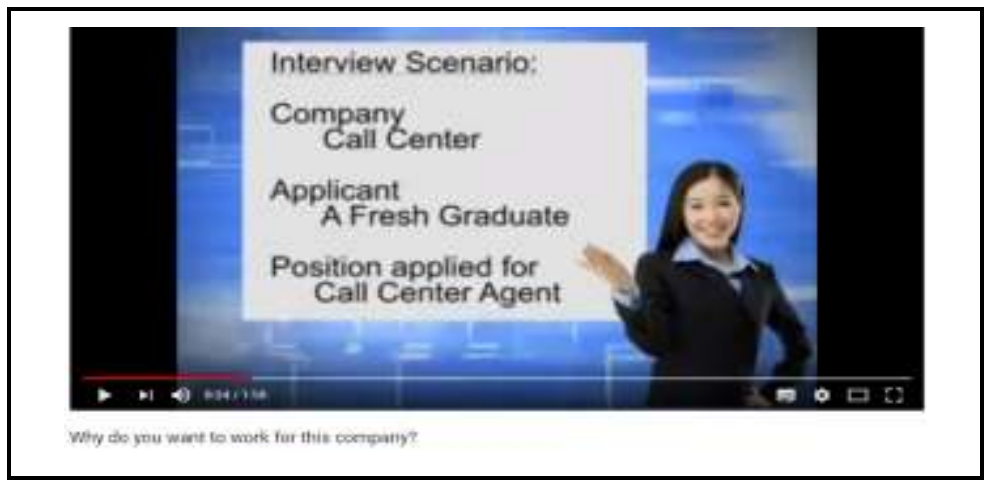

\subsection{Redundancy Principle}

Based on this principle, people learn more deeply from graphics and narration than from graphics, narration, and on-screen text. It is argued that redundancy of media presentation may waste precious processing capacity by trying to reconcile the two verbal streams of information or have students focus on the printed words rather than the relevant portions of the graphics. In this study, this principle appears not to match with the students' actual conditions. As EFL learners, most of them were not proficient enough to rely on graphics and mere auditory messages. The specialized words and diction in the videos together with the students' nonnative background seem to make the learning process deviate from this redundancy principle. The use of on-screen text provided by either the videos themselves or Youtube's optional closed-captioning feature can often help the students to make a connection between what was said and what was written/captioned.

Figure 5: On-screen text

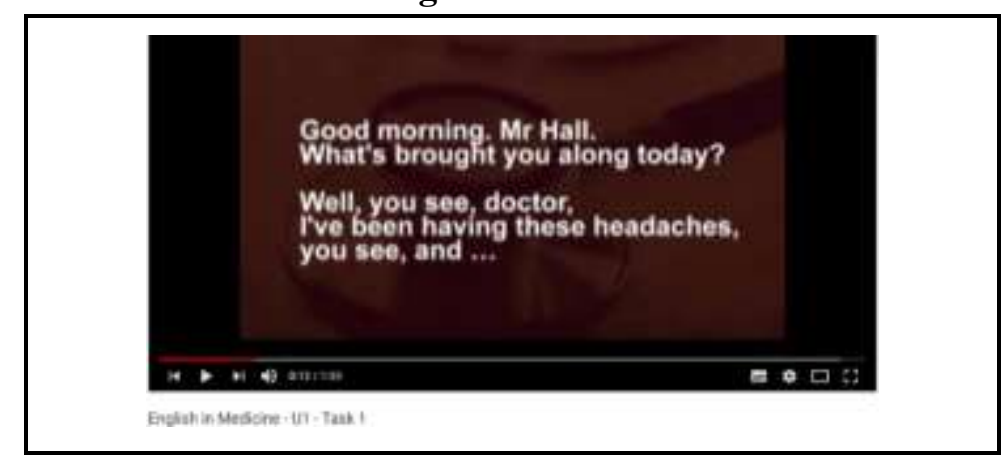


Secondly, the nature of video demonstration in this study does not depict complicated process requiring step-by-step comprehension. Instead, it consists of demonstration of answering job-interview questions, medical examination, or presentation in English along with samples of use of linguistics features of the discourse. Thus, processing of more than two streams of information appears not to cause information processing overload.

\subsection{Spatial Contiguity Principles}

People learn better from multimedia message when corresponding words and graphics are presented near rather than far from each other on the screen. Spatial contiguity arguably helps learners build connection between corresponding words and graphics. This principle is facilitated with ease due to feature of captions or subtitles on Youtube. Students could therefore monitor their comprehension by making meaning of these two modes of communication, visual from the moving picture and subtitles and auditory from the narration.

Figure 6: Youtube's closed-captioning (CC) feature

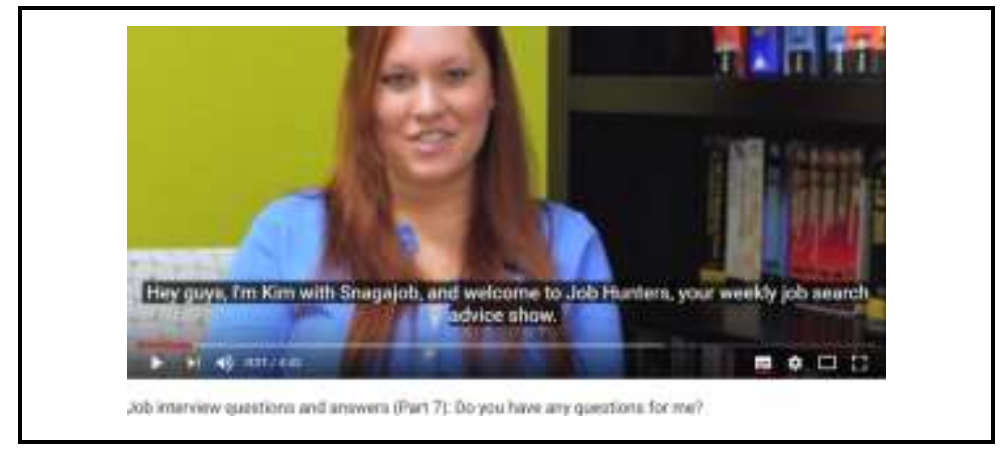

\subsection{Temporal Contiguity Principles}

People learn from multimedia messages when corresponding graphics and narration are presented simultaneously rather than successively. Like spatial contiguity, this principle helps learners build connection between corresponding words and graphics. The video material depicts contextual situation and demonstration of both physical and language performances. Naturally, in the online videos, these visual and auditory modes of communication are presented simultaneously. Moving pictures along with narration or subtitles fulfill the information processing principles as proposed by Mayer (2014).

\section{Conclusion}

This study argues that principles of multimedia learning and multimedia processing allow more multimodal exposures to enhance language learning in terms of learning contexts, target culture understanding, and language function practice. It is concluded that audio-visual instructional media potentially empower teachers to facilitate more effective learning process in the class by engaging learners in the media. The elaboration of audio and visual media appears to increase student motivation as the nature of foreign language learning demands more than merely textual information.

More studies are required to gain more comprehensive measure of to what extend multimodal material effectively influences language skill practice in EFL contexts. Another opportunity for research could be addressed towards investigating how principles of multimedia processing work for various language learning purposes. 


\section{References}

Al-Khayyat, A. S. J. 2016. The impact of audio-visual aids (AVA) and computerize materials (CM) on university ESP students' progress in English language. International Journal of Education and Research, 4(1), 273-282

Applying Science of Learning in Education.

Ashaver, D. \& Igyuve, S. M. 2013. The use of audio-visual materials in the teaching and learning processes in colleges of education in Benue state-Nigeria. IOSR Journal of Research \& Method in Education, 1(6), 44-55.

Bajrami, L. \& Ismaili, M. 2016. The role of video materials in EFL classrooms. International Conference on Teaching and Learning English as an Additional Language. Antalya: Glob ELT.

Berk, R. A. 2009. Multimedia teaching with video clips: TV, movies, YouTube, and $\mathrm{mtv} U$ in the college classroom. International Journal of Technology in Teaching and Learning, 5(1), 1-21

Bruti, S. 2015. Teaching learners how to use pragmatic routines through audio-visual material. In Fortanet-Gómez, I \& Camiciottoli, B. C (eds.). Multimodal analysis in academic settings: From research to teaching. New York: Routledge, 213-237

Fortanet-Gómez, I. \& Camiciottoli, B. C. 2015. Multimodal analysis in academic settings: From research to teaching. New York: Routledge.

Matthiessen, C. M. I. M. 2007. The multimodal page: A systemic functional exploration. In Royce, T. D \& Bowcher, W. L (eds.). New directions in the analysis of multimodal discourse. Mahwah, NJ: Lawrence Erlbaum Associates, Inc., 1-14.

Mayer, R. E. 2009. Multimedia learning. New York: Cambridge University Press.

Mayer, R. E. 2014. Research-based principles for designing multimedia instruction.

Mishra, S \& Sharma, R. 2005. Interactive multimedia and education training. London: Idea Group Inc.

O’Halloran, K. L. 2004. Multimodal discourse analysis. London: Continuum.

Reddi, U.V. 2003. Multimedia as an educational tool. In Reddi, U. V \& Mishra, S (eds.). Educational multimedia: A handbook for teacher-developers. New Delhi: CEMCA

Rezaie, S. H. S. \& Barani, G. 2011. Iranian teachers' perspective of the implementation of audiovisual devices in teaching. Procedia Computer Science, 3(2011), 1576-1580.

Rusmiati \& Rosdiana. 2017. The role of audio visual aids in EFL classroom. Proceedings of the 1st International Conference on Innovative Pedagogy. Banda Aceh: STKIP Bina Bangsa Getsempena. 